\title{
Women living with ovarian cancer described changes in day to day living, major challenges, and sources of support
}

Howell D, Fitch MI, Deane KA. Impact of ovarian cancer perceived by women. Cancer Nurs 2003;26:1-9.

\section{QUESTION: What are the perceptions of women living with ovarian cancer?}

Design

Qualitative study.

\section{Setting}

Toronto, Ontario, Canada.

\section{Participants}

18 women (age range 35-73 y) with ovarian cancer were identified through 2 major cancer centres and a local ovarian cancer support group.

\section{Methods}

Data were collected through 1 hour semistructured telephone interviews. Questions related to the effects of ovarian cancer on day to day living, major challenges, and sources of support. Interview data were transcribed verbatim and reviewed by the 3 authors, who identified themes arising from an inductively derived coding scheme.

\section{Main findings}

(1) Effect of cancer on day to day living. Cancer profoundly altered women's daily lives, resulting in the loss of usual activities, inability to work, and financial concerns arising because of uncertainty about future health needs and employment. Women struggled to integrate changes and experienced emotional distress as they realised that life was changed forever. The diagnosis of ovarian cancer had an impact on the family. Women described changing roles and fears for husbands. The marital relationship became strained as husbands adopted new roles and responsibilities to support their wives and maintain the household. Unspoken fears affected communication, and women worried about the effects of added stress on their husbands' health. Altered sexuality occurred because of treatment related physical changes, which created discomfort that compromised intimate relations. The purpose of sexual relations was altered, given changing prospects for future childbearing. Women expressed worries about children, had difficulties talking about cancer with them, and feared the increased risk of ovarian cancer in female children. Those with young children were particularly fearful for the future, whereas those with adult children found their care and support to be invaluable.

(2) Major challenges. Living with uncertainty was a challenge, and dismal survival statistics were a constant reminder of the uncertainty of the future. Women felt a lack of control over events in their lives, which led to a profound sense of sadness and fear that was difficult to share with others. They were concerned about becoming dependent on others and had a fear of the unknown, as they were acutely aware of the possibility of death. A second challenge was the stigma of cancer. Women sensed the discomfort of others and felt that people avoided them because they did not know how to be with a person with cancer. A third challenge was facing death. The late stage of disease at diagnosis meant that most women were aware of the potential for death when they were diagnosed. Thoughts of leaving young children and not being with them as they grew up were particularly difficult.

(3) Sources of support. Women described support from various sources. Extended family members provided emotional and practical support. Some women found that friends in the community, personal relationships, and the church community were especially helpful during the treatment phase.

\section{Conclusions}

Women living with ovarian cancer described radical changes in daily living and the effects on their families including changing roles and fears for husbands, altered sexuality, and worries about children. Major challenges were living with uncertainty, the stigma of cancer, and facing death. Sources of support included extended family, personal relationships, and church communities.

\section{COMMENTARY}

Howell et al report the findings of a qualitative study in 2 separate reports. The findings support existing literature on theoretical aspects of coping with threat, uncertainty, and chronicity. ${ }^{1}$ The authors indirectly refer to this scientific context, but do not explicitly place the findings of this study into such a framework. The common human responses the authors describe are consistent with the responses of people dealing with a chronic, life threatening illness. ${ }^{1}$

The authors used semistructured interviews, in which participants responded to specific questions without additional probing. The topics explored were clearly identified. Telephone interviews were appropriate because the authors determined there was no need to assess non-verbal behaviour to give cues for further questioning: ${ }^{2}$ this format also reduced inconvenience for participants. When researchers gather sensitive information, the anonymity of the telephone may facilitate collection of data.

18 women were interviewed to explore perceptions of living with ovarian cancer; 11 of these participants who had recurrences were interviewed to explore perceptions of cancer recurrence. This sample size is large for a qualitative study and would have resulted in a large amount of data for analysis. The authors presented fairly long quotations, although it is unclear whether these quotations represented responses from several participants or from one person.

The findings presented in these 2 reports can assist nurses by identifying themes to be addressed when caring for women with ovarian cancer. Participants reported that once they were diagnosed with a relapse, nurses treated them differently. In most cases, nurses realise that a relapse means that a patient cannot be cured and that treatment will be directed at control or palliation. Patients, on the other hand, usually still think in terms of cure. For nurses, this difference creates a dilemma. Nurses do not want to enter the physician's realm by clarifying the issue for the patient, and many are uncomfortable when confronted with the inevitability of a patient's death. Maintaining hope in patients requires that the "object" of hope change from "cure" to whatever a patient will hope for. This change in what is hoped for is reflected in the comments of participants in this study. By attending to these comments, nurses may gain insight into how they might communicate with patients in this situation. 


\title{
Women with ovarian cancer described waiting for recurrence, facing the diagnosis of recurrence, managing treatment related concerns, and attempting to regain control
}

\author{
Howell D, Fitch MI, Deane KA. Women's experiences with recurrent ovarian cancer Cancer Nurs 2003;26:10-17. \\ QUESTION: How do women with ovarian cancer experience cancer recurrence?
}

Design

Qualitative study.

\section{Setting}

Toronto, Ontario, Canada.

\section{Participants}

18 women (age range $35-73$ y) with ovarian cancer were identified through 2 major cancer centres and a local ovarian cancer support group.

\section{Methods}

Data were collected through 1 hour semistructured telephone interviews. Questions related to diagnosis and treatment, treatment decisions, acquisition of information and supportive care, effects of illness, and lifestyle changes. Interview data were transcribed verbatim and reviewed by the 3 authors, who identified themes arising from an inductively derived coding scheme.

\section{Main findings}

4 main themes emerged: waiting for recurrence, facing the diagnosis of recurrence, managing treatment related concerns, and attempting to regain control. (1) Waiting for recurrence. Most women were aware of the risk of recurrence at the time of diagnosis, having learnt about survival rates from books, pamphlets, the internet, physicians, and other patients. Women had mixed feelings about knowing these statistics at a time when they were trying to deal with their cancer diagnosis and treatment. Some did not want to hear the statistics because they caused fear and anxiety, particularly regarding recurrence at follow up. However, foreknowledge of the possibility did not prepare women for news of a recurrence, which evoked feelings of being overwhelmed, devastated, and shocked. (2) Facing the diagnosis of recurrence. Some women had little time between initial diagnosis and recurrent disease, and thus little reprieve from ongoing treatment and side effects. Periods of watchful waiting and monitoring tumour markers such as CA-125 concentrations were difficult. Increasing CA-125 concentrations led to panic as they signalled cancer recurrence. (3) Managing treatment related concerns. Women perceived that care providers treated them differently when cancer recurred. They felt that communication became strained, which they interpreted to mean that they were without hope, and led to feelings of hopelessness. Women felt that information about treatment options was more difficult to obtain after recurrence, and that they had to push for further information. Some women changed physicians to obtain the care they desired, whereas others felt limited to specific treatments because of geography and lack of access to other physicians. Overall, women felt that few treatment options were available when cancer recurred, and that the focus changed from prolongation of life to symptom management. Oncology nurses were seen as a source of support and understanding. The need for involvement and control in treatment decisions increased as women completed first line treatment, faced recurrence, and improved their understanding of ovarian cancer and their bodies. To gain some control over their progressing disease, some women requested second opinions or complained to the hospital administration. (4) Attempting to regain control. With the recurrence of cancer, women were desperate to find a treatment to contain or delay tumour growth. 12 women used alternative therapies, such as therapeutic touch or herbal remedies, which helped them gain a sense of control over their disease.

\section{Conclusion}

Women with ovarian cancer described waiting for recurrence, facing the diagnosis of recurrence, managing treatment related concerns, and attempting to regain control.

COMMENTARY - continued from previous page

Both reports clearly showed that having ovarian cancer affected every aspect of life. The diagnosis seemed to magnify areas of vulnerability, and women found they did not always have the resources to cope. Nursing interventions should prepare women to use their current means of coping and to expand coping skills. Nurses can help patients to reappraise their individual situations to better reflect reality. For example, for many women, ovarian cancer can best be regarded as a chronic illness that will extend over time, and which can, to some degree, be controlled. Through understanding predictable aspects of the disease and how to manage symptoms and side effects, women can gain an increased sense of personal control, which in turn may decrease distress. Nurses can also teach stress reduction techniques to help women control the anxiety that inevitably accompanies medical monitoring. Such techniques could also help in the management of pain, nausea, and other side effects of treatment. ${ }^{3}$ Nurses and patients can select specific outcomes collaboratively and work to achieve them by focusing on education, developing communication skills within the family, finding sources of personal and financial support, and rebuilding confidence and a sense of physical and emotional integrity.

Kathleen Sodergren, RN, MSN, PhD Breast Cancer Clinical Specialist Mercy and Unity Hospitals Fridley, Minnesota, USA

1 Jalowiec A. Coping with illness: synthesis and critique of the nursing coping literature from 1980-1990. In Barnfather JS, Lyon BL, editors. Stress and coping: state of the science and implications for nursing theory, research and practice. Indianapolis: Center Nursing Press of Sigma Theta Tau Inte

Funk SG, Torquist EM, Champagne MT, et al, editors. Key aspects of comfort: management of pain, fatigue, and nausea. New York: Springer, 1989. 